\title{
Computational fluid dynamics using in vivo ultrasound blood flow measurements
}

Traberg, Marie Sand; Pedersen, Mads Møller; Hemmsen, Martin Christian; Nielsen, Michael Bachmann; Jensen, Jørgen Arendt

\section{Published in:}

Proceedings of IEEE International Ultrasonics Symposium

Publication date:

2012

Link back to DTU Orbit

Citation (APA):

Traberg, M. S., Pedersen, M. M., Hemmsen, M. C., Nielsen, M. B., \& Jensen, J. A. (2012). Computational fluid dynamics using in vivo ultrasound blood flow measurements. In Proceedings of IEEE International Ultrasonics Symposium IEEE.

\section{General rights}

Copyright and moral rights for the publications made accessible in the public portal are retained by the authors and/or other copyright owners and it is a condition of accessing publications that users recognise and abide by the legal requirements associated with these rights.

- Users may download and print one copy of any publication from the public portal for the purpose of private study or research.

- You may not further distribute the material or use it for any profit-making activity or commercial gain

- You may freely distribute the URL identifying the publication in the public portal

If you believe that this document breaches copyright please contact us providing details, and we will remove access to the work immediately and investigate your claim 


\title{
Computational Fluid Dynamics Using In Vivo Ultrasound Blood Flow Measurements
}

\author{
Marie Sand Enevoldsen ${ }^{1}$, Mads Møller Pedersen ${ }^{2}$, Martin Christian Hemmsen ${ }^{1}$, \\ Michael Bachmann Nielsen ${ }^{3}$ and Jørgen Arendt Jensen ${ }^{1}$ \\ ${ }^{1}$ Center for Fast Ultrasound Imaging, Dept. of Elec. Eng., Bldg. 349, Technical University of Denmark, \\ DK-2800 Lyngby, Denmark \\ 2 Dept. of Radiology, Gentofte University Hospital, DK-2900 Hellerup, Denmark \\ ${ }^{3}$ Dept. of Radiology, Copenhagen University Hospital, Rigshospitalet, DK-2100 Copenhagen, Denmark
}

\begin{abstract}
This paper presents a model environment for construction of patient-specific computational fluid dynamic (CFD) models for the abdominal aorta (AA). Realistic pulsatile velocity waveforms are employed by using in vivo ultrasound blood flow measurements. Ultrasound is suitable for acquisition of blood velocity profiles, but these are influenced by noise, which will cause convergence problems in CFD simulations. Therefore, physiological smoothing of the velocity profiles is needed. This paper uses the Womersley-Evans model for physiological smoothing of measured blood velocity profiles in the AA. The geometry for the CFD simulation model was obtained by segmentation of MRI scans using a 3 Tesla scanner (Magnetom Trio, Siemens Healthcare, Erlangen, Germany). Spectral velocity data were obtained from a BK Medical ProFocus scanner using a research interface. All data were obtained from healthy volunteers. The estimated and smoothed velocity profiles were quantitatively compared. The energy contained in the velocity profile after smoothing is $65 \%$ larger relative to the noise contaminated estimated profiles. In conclusion, a model environment that produces realistic patient-specific CFD simulation models without convergence issues has been developed. The data processing for the model environment can be performed within six hours which is fast enough to be used in the clinical setting.
\end{abstract}

\section{INTRODUCTION}

Computational fluid dynamic (CFD) simulations are valuable for studying blood flow pattern changes, which are thought to play an important role in the pathogenesis of abdominal aortic aneurysms (AAA) [1], [2]. The purpose of this paper is to present a model environment that allows for construction of patient-specific CFD models for investigation of AAA.

Ultrasound is suitable for acquisition of patient-specific blood velocity profiles, however, these profiles are influenced by noise, which will cause convergence problems in the CFD simulations. Therefore, physiological smoothing is needed. Here it is proposed that the Womersley-Evans model [3] is suitable for physiological smoothing. The model environment is developed using the healthy abdominal aorta (AA) as an example.

There are numerous studies on CFD simulations of blood flow in the AA, and the challenge is to provide realistic boundary conditions (BCs) for the inlet and outlet. Di Martino et al. [1] utilized averaged blood flow velocity from the AA obtained by colour Doppler measurements. The inlet profile was assumed to be parabolic (fully developed). However, this is not the case in vivo as shown by Moore et al. [4] and $\mathrm{Ku}$ [5]. Figueroa et al. [6] obtained the volume flow rate for an AA using magnetic resonance imaging (MRI) and performed CFD simulations with a time-varying parabolic profile. The time-varying velocity was derived using the principles stated by Womersley [7]. None of these studies quantified how well the inlet condition in the CFD simulation models corresponds to the in vivo situation.

In this work the physiologically smoothed profiles are compared to estimated in vivo profiles both visually and quantitatively, and it is hypothesised that the Womersley-Evans model reproduce smooth realistic inlet profiles, which can be implemented in CFD simulation models.

\section{Methods}

A patient-specific CFD simulation model environment was constructed. A flow diagram of the model environment is displayed in Fig. 1. As an example, a CFD simulation model for a 23 years old healthy male volunteer is presented here. This study was approved by the Danish National Committee on Biomedical Research Ethics (H-3-2009-102, 2009/09/01).

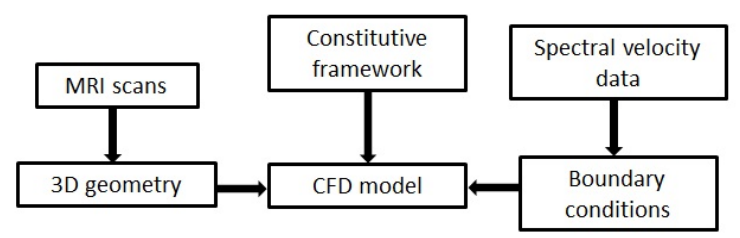

Fig. 1: Flow diagram of the model environment.

\section{A. Magnetic resonance imaging and segmentation}

The lumen geometry of the AA was obtained by segmentation of a 3D magnetic resonance angiography (Magnetom Trio, Siemens Healthcare, Erlangen, Germany). The imaging sequence applied for the MRI procedure was the Native True FISP (Fast Imaging with Steady-state Precession) [8]. This 
sequence enables imaging of arteries without the use of intravascular contrast agents.

The segmentation of the MRI scan was performed using ScanIP (Simpleware ltd., Exeter, United Kingdom). The geometry included the iliac bifurcation and was cut just below the branching of the renal arteries. The segmentation procedure was semi-automatic including three consecutive steps: (1) Initial automatic coarse segmentation , (2) Manual editing of the coarse segmentation, and (3) Smoothing of the segmented AA lumen.

\section{B. Spectral velocity data}

Five sets of spectral velocity data each lasting five seconds were acquired from the volunteer, see Fig. 2. A convex array transducer (BK8803, BK Medical, Herlev, Denmark) connected to a 2202 ProFocus scanner (BK Medical, Herlev, Denmark) equipped with a UA2227 research interface [9] were used. The scan sequence was controlled by the dedicated grabber program "CFU Data Grabber" [9]. The CFU Grabber GUI enables acquisition of raw data directly from the scanner, and download of the raw data to a hard drive. To obtain usable data the pulsing strategy was designed such that the two types of data, i.e. the B-mode image lines and the spectral velocity data were acquired interleaved. After acquisition, the data were sorted and processed off-line using customized routines developed for MATLAB (Mathworks, Natick, MA, USA). The spectral velocity data were needed for estimation of velocity profiles as function of time and depth to be used as the inlet condition in the CFD simulation model.

\section{Velocity estimator}

The velocity estimator developed for this work is based on the time shift between consecutively received flow signals. When the time between consecutively received signals and the propagation speed of ultrasound in the tissue are known, the velocity of the pulsating blood can be calculated as [3]

$$
v_{z}=\frac{c}{2} \frac{t_{s}}{T_{p r f}},
$$

where $v_{z}$ is the axial velocity component, $c=1540 \mathrm{~m} / \mathrm{s}$ is the propagation speed of ultrasound in blood, $t_{s}$ is the time shift between received signals due to the movement of the blood scatters, and $T_{p r f}$ is the pulse repetition time. The time shift between received signals was estimated using the cross-correlation function as suggested by a number of authors [10]-[12]. In this work the estimated cross-correlation functions were improved by averaging over 128 received signals. Additionally, the time associated with the maximum in the cross-correlation was improved by interpolating around the maximum peak. A second order polynomial was fitted to the three points around the maximum of the cross-correlation function, and then the time shift was determined from the position of the maximum of the polynomial [12].

The velocity variation over time was estimated at all depths and compared to the velocity profile resulting from physiological smoothing by the Womersley-Evans model.

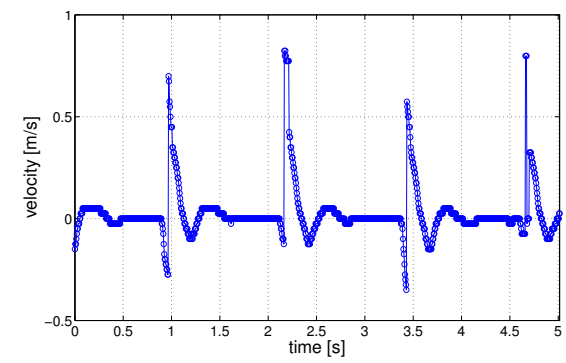

Fig. 2: Estimated velocity variation using the time-shift approach in the centre of the abdominal aorta.

\section{Womersley-Evans model}

Physiological smoothing was obtained by decomposing the average flow velocity in the center of the AA into ten harmonics and then applying the Womersley-Evans model [7], [13] to reconstruct smooth velocity profiles, which are continuous in both time and space. Mathematically, under the assumption that the pulsation is periodic, the velocity waveform can be decomposed into a Fourier serie [14] and then added to the parabolic profile characterizing the fully-developed flow in a stiff pipe. Womersley [7] found the relation between the flow rate and the pressure for each harmonic component of the velocity Fourier series. Evans [13] provided the method for determination of the velocity profile, when the flow rate is known. Neglecting entrance effect, the temporal-spatial velocity profile was determined as [2], [3],

$$
\begin{aligned}
v(t, r)= & 2 v_{0}\left(1-\left(\frac{r}{R}\right)^{2}\right) \\
& +\sum_{m=1}^{10}\left|V_{m}\right|\left|\psi_{m}\right| \cos \left(m \omega t-\phi_{m}+\chi_{m}\right),
\end{aligned}
$$

where $v_{0}$ is the mean velocity in the AA, $R$ is the total AA radius, $V_{m}$ is the sinusoidal components resulting from the Fourier decomposition, $\psi_{m}$ is a function describing how the velocity changes with time and position during one period for each sinusoidal component, $\phi_{m}$ is the phase of the sinusoids, and $\chi_{m}$ is the phase of $\psi_{m}$. The function $\psi_{m}$ is given as,

$$
\psi_{m}\left(\tau_{m}, r\right)=\frac{\tau_{m} J_{0}\left(\tau_{m}\right)-\tau_{m} J_{0}\left(\tau_{m} \frac{r}{R}\right)}{\tau_{m} J_{0}\left(\tau_{m}\right)-2 J_{1}\left(\tau_{m}\right)},
$$

where $J_{n}$ are the $n$ 'th order Bessel functions of first kind, and $\tau_{m}=j^{2 / 3} \alpha_{m}$. The Womersley number $\alpha_{m}$ is defined as,

$$
\alpha_{m}=R \sqrt{\frac{\rho_{\text {blood }}}{\mu_{\text {blood }}} \omega_{m}},
$$

where $\rho_{\text {blood }}=1,060 \mathrm{~kg} / \mathrm{m}^{2}$ is the mass density of blood and $\mu_{\text {blood }}=3.5 \mathrm{mPa} \cdot \mathrm{s}$ is the viscosity of blood assuming that it is a Newtonian fluid. This assumption is valid for large systemic arteries [15], [16]. 


\section{E. CFD simulation model}

The AA geometries were loaded into the commercial finite element software COMSOL Multiphysics (COMSOL AB, Stockholm, Sweden) using the CFD module. The geometry was meshed by the build-in meshing algorithm in COMSOL Multiphysics and consisted of 213,591 tetrahedral elements. The applied constitutive framework for the CFD simulations was the Navier-Stokes equation assuming that blood is an incompressible isotropic Newtonian fluid,

$$
\rho_{\text {blood }}\left(\frac{\partial \vec{v}}{\partial t}+(\vec{v} \cdot \nabla) \vec{v}\right)=-\nabla p+\mu_{\text {blood }} \nabla^{2} \vec{v}+\rho_{\text {blood }} \vec{g}
$$

where $\vec{v}$ is the velocity field, $\nabla$ is the vector differential operator, $\nabla p$ is the pressure gradient, $\nabla^{2}$ is the Laplacian, and $\vec{g}$ is gravity.

\section{RESULTS}

In Fig. 3 the estimated in vivo velocity variation over one cardiac cycle is compared to the same velocity variation after physiological smoothing. The estimated velocity variation is heavily influenced by noise. However, the two velocity curves are comparable.

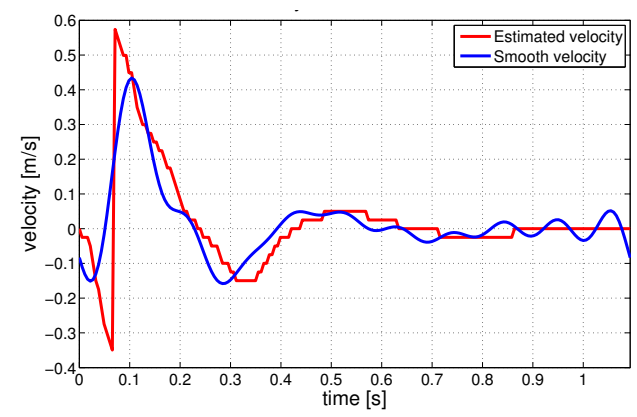

Fig. 3: Comparison of the velocity variation over one cardiac cycle before (indicated by the solid red line) and after (indicated by the blue solid line) physiological smoothing using the Womersley-Evans model.

The comparison is quantified by calculating the difference in energy between the two curves and comparing to the energy contained in the curve after smoothing. This is done by,

$$
E_{s}=\sum_{i=1}^{N_{d}} \sum_{j=1}^{N_{s}}|v(i, n)|^{2}
$$

where $E_{s}$ is the energy of the signal $v(i, n), N_{d}$ is the number of samples in depth, $N_{s}$ is the number of samples in one period, and $v(i, n)$ is the velocity variation as a function of time at a given depth. The energy of the smooth velocity curve in the centre of the AA is $E_{s}=285 \mathrm{~J}$ compared to $E_{s}=$ $172 \mathrm{~J}$ for the estimated noise-contaminated curves. Thus, the curve after smoothing contains $66 \%$ more energy relative to the noise-contaminated curve prior to smoothing.

Fig. 4 shows the velocity as a function of both time and space obtained from estimation of raw spectral velocity data,

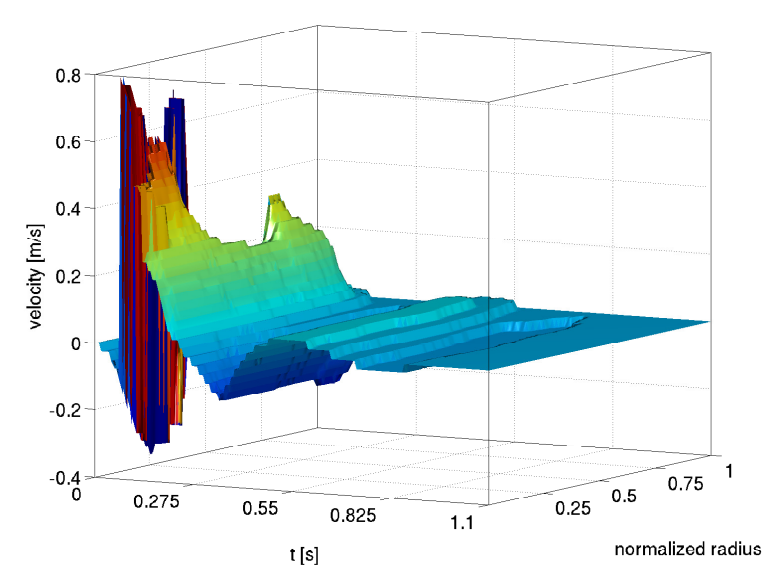

Fig. 4: Estimated velocity profile as a function of both time and depth in the abdominal aorta based on in vivo data.

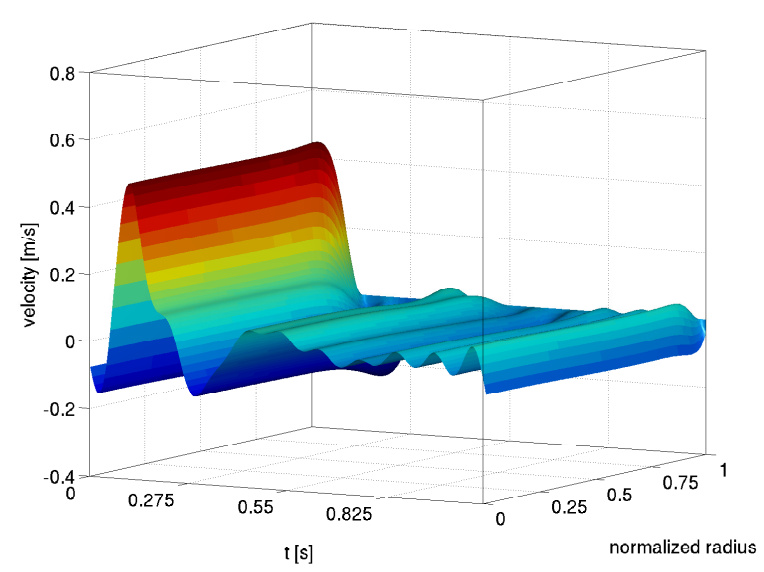

Fig. 5: Velocity profile as a function of time and depth in the abdominal aorta after physiological smoothing.

as data are a acquired across the entire AA diameter. It is observed that the measurements are influenced by noise, especially when the velocity gradients are high in the beginning of the cardiac cycle.

In Fig. 5 the velocity as a function of time and space is displayed after physiological smoothing. Comparing this profile to the one in Fig. 4 the smoothing compensates for the discontinuities in the estimated velocity. The smooth velocity profile is comparable to a plug flow. The peak velocity after smoothing is lower, $0.5 \mathrm{~m} / \mathrm{s}$, compared to the peak velocity of $0.75 \mathrm{~m} / \mathrm{s}$ before smoothing.

The smooth velocity profiles were applied directly as inlet condition for the CFD simulation model. The CFD simulation model converges when the standard solver algorithm in COMSOL Multiphysics is applied. Fig. 6 shows the results.

The computation time in this case is 57 minutes. As it can be seen from Fig. 6 the geometry as well as the inlet condition influence the blood flow pattern in the AA. At the peak systole (Fig. 6a) an almost laminar flow pattern is seen, but when the blood decelerates in the early diastole (Fig. 6b) disturbances 
in the laminar flow pattern are seen.

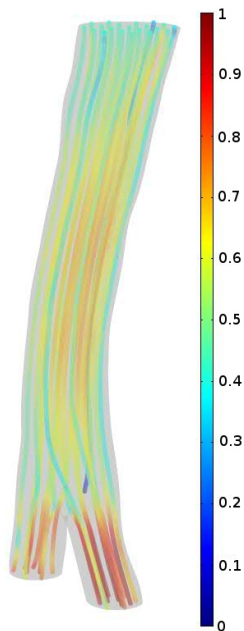

(a)

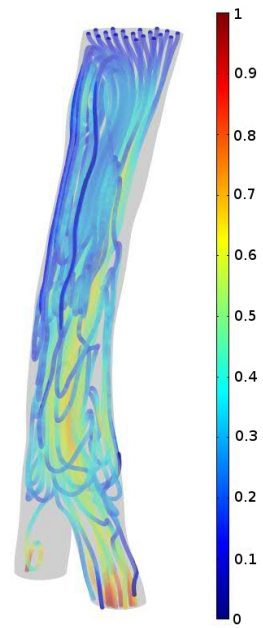

(b)
Fig. 6: CFD simulation results of the velocity streamlines in the AA lumen. Panel (a) shows the velocity streamlines at peak systole in the cardiac cycle. Panel (b) shows the velocity streamlines in the early diastole of the cardiac cycle. Note that the colourbar indicate the normalized velocity.

\section{Discussion AND CONCLUSION}

Investigations of blood flow patterns using CFD simulation models are numerous in the literature today. This study has developed a model environment for construction of patientspecific CFD simulation models for the AA that ensures convergence, and gives realistic results. This is due to the physiological smoothing of the in vivo estimated velocity profiles. No one have quantified the validity of using the WomersleyEvans model for physiological smoothing. This paper presents such a quantification. The estimated in vivo velocity profile is directly compared to the velocity profile after physiological smoothing by calculation of the relative increase in energy after smoothing. The smooth velocity profile contains $66 \%$ more energy compared to the noise-contaminated estimated profile. But the energy content after smoothing is $116 \%$ larger compared to the energy difference between the two. Therefore, it is concluded that the Womersley-Evans model is a suitable method for obtaining smooth continuous profiles, which can be applied in CFD simulation models.

The velocity profile in the AA after smoothing resembles a plug flow, and this observation corresponds with results from $\mathrm{Ku}$ et al. [5]. However, when performing physiological smoothing it is important to not smooth to much, as this will smooth out the steep velocity gradients in cardiac cycle.

To establish a model environment that is usable in the clinical setting it is important that the computation time of both the velocity estimation, physiological smoothing, image segmentation, and CFD simulations are within a reasonable time frame. The computation time for velocity estimation and physiological smoothing is within minutes. The image segmentation is largely dependent on the image quality and the skills of the operator. The computation time for the CFD simulation presented here is 57 minutes when performed on a standard PC (Dell Precision T5500, Intel Xeon Quad Core with $4 \mathrm{~GB}$ ram). Combined the process can be performed within six hours, thus it is fast enough to be used in the clinic setting.

\section{ACKNOWLEDGMENT}

This work was supported by project 55562 at Technical University of Denmark, Radiometer Medical ApS., and the Danish Ministry of Science, Technology, and Innovation.

\section{REFERENCES}

[1] E. Di Martino, G. Guadagni, A. Fumero, G. Ballerini, R. Spirito, P. Biglioli, and A. Redaelli, "Fluid-structure interaction within realistic three-dimensional models of the aneurysmatic aorta as a guidance to assess the risk of rupture of the aneurysm," Medical engineering \& physics, vol. 23, no. 9, pp. 647-655, 2001.

[2] Y. Papaharilaou, J. Ekaterinaris, E. Manousaki, and A. Katsamouris, "A decoupled fluid structure approach for estimating wall stress in abdominal aortic aneurysms," Journal of Biomechanics, vol. 40, no. 2, pp. 367-377, 2007.

[3] J. A. Jensen, Estimation of Blood Velocities Using Ultrasound: A Signal Processing Approach. New York: Cambridge University Press, 1996.

[4] J. Moore Jr, S. Maier, D. Ku, and P. Boesiger, "Hemodynamics in the abdominal aorta: a comparison of in vitro and in vivo measurements," Journal of Applied Physiology, vol. 76, no. 4, pp. 1520-1527, 1994.

[5] D. Ku, "Blood flow in arteries," Annual Review of Fluid Mechanics, vol. 29, no. 1, pp. 399-434, 1997.

[6] C. Figueroa, I. Vignon-Clementel, K. Jansen, T. Hughes, and C. Taylor, "A coupled momentum method for modeling blood flow in threedimensional deformable arteries," Computer Methods in Applied Mechanics and Engineering, vol. 195, no. 41-43, pp. 5685-5706, 2006.

[7] J. R. Womersley, "Oscillatory motion of a viscous liquid in a thin-walled elastic tube. I: The linear approximation for long waves," Phil. Mag., vol. 46, pp. 199-221, 1955.

[8] P. Weale, "syngo Native - Non Contrast MR Angiography Techniques," 2011, http://www.clinical-mri.com/pdf/IT/hardware/MRA_Native_final. pdf.

[9] M. C. Hemmsen, S. I. Nikolov, M. M. Pedersen, M. J. Pihl, M. S. Enevoldsen, J. M. Hansen, and J. A. Jensen, "Implementation of a versatile research data acquisition system using a commercially available medical ultrasound scanner," IEEE Trans. Ultrason., Ferroelec., Freq. Contr., vol. 59, no. 7, pp. 1487-1499, 2011.

[10] D. Dotti, E. Gatti, V. Svelto, A. Uggè, and P. Vidali, "Blood flow measurements by ultrasound correlation techniques," Energia Nucleare, vol. 23, pp. 571-575, 1976.

[11] O. Bonnefous, P. Pesqué, and X. Bernard, "A new velocity estimator for color flow mapping," in Proc. IEEE Ultrason. Symp., 1986, pp. 855-860.

[12] S. G. Foster, P. M. Embree, and W. D. O'Brien, "Flow velocity profile via time-domain correlation: Error analysis and computer simulation," IEEE Trans. Ultrason., Ferroelec., Freq. Contr., vol. 37, pp. 164-175, 1990.

[13] D. H. Evans, "Some aspects of the relationship between instantaneous volumetric blood flow and continuous wave Doppler ultrasound recordings III," Ultrasound Med. Biol., vol. 8, pp. 617-623, 1982b.

[14] G. Truskey, F. Yuan, and D. Katz, Transport phenomena in biological systems. Pearson Prentice Hall Upper Saddle River, NJ, 2004.

[15] P. Rissland, Y. Alemu, S. Einav, J. Ricotta, and D. Bluestein, "Abdominal aortic aneurysm risk of rupture: patient-specific fsi simulations using anisotropic model,' Journal of Biomechanical Engineering, vol. 131, pp. 031 001-1-013001-10, 2009.

[16] C. Scotti, J. Jimenez, S. Muluk, and E. Finol, "Wall stress and flow dynamics in abdominal aortic aneurysms: finite element analysis vs. fluid-structure interaction," Computer Methods in Biomechanics and Biomedical Engineering, vol. 11, no. 3, pp. 301-322, 2008. 